\title{
INVESTIGACIONES
}

\section{Preferencias académicas y laborales en la adolescencia: Una perspectiva de género}

\author{
Academic and working preferences in adolescence: A gender perspective \\ Preferências académicas e profissionais na adolescência: \\ Uma perspectiva de género
}

María-Carmen Ricoy, ${ }^{a}$ Cristina Sánchez-Martínez

${ }^{a}$ Facultad de Ciencias de la Educación, Universidad de Vigo, España

Telf.: 988387065. Correo electrónico: cricoy@uvigo.es

\section{RESUMEN}

Es importante que en la adolescencia puedan trazar de forma consciente su recorrido vital para afianzar paulatinamente sus aspiraciones académicas y laborales, de las que debe ser conocedor el profesorado y progenitores. En este trabajo, se trata de descubrir las preferencias académicas y profesionales del alumnado del último curso de la educación obligatoria en España. La investigación se ha abordado a través de la vertiente cualitativa, a partir de un estudio de casos múltiples. Como resultados y conclusiones cabe resaltar que se detectan diferencias por género en cuanto a la inclinación revelada por el alumnado hacia asignaturas de ciencias experimentales o de "lenguas". Los y las adolescentes se decantan principalmente por las profesiones relacionadas con el sector servicios.

Palabras clave: adolescentes, educación secundaria obligatoria, preferencias académicas, expectativas laborales.

\section{ABSTRACT}

It is important for teens during adolescence to plan conscientiously their vital route to gradually strengthen academic and career aspirations, and this must be known by teachers and progenitors. The aim of this paper is to discover the academic and working preferences of students in their final year of compulsory education in Spain. The investigation was developed through qualitative analysis of multiple case studies. As results and conclusions, it should be noted that differences by gender were detected regarding the inclination shown by students towards experimental sciences and language subjects. Teens opt for professions mainly related to services sector.

Key words: adolescents, compulsory secondary education, academic preferences, job expectations. 


\section{INTRODUCCIÓN}

El fracaso y abandono de los estudios obligatorios, post-obligatorios y superiores que se produce especialmente en los primeros años, continúa constituyendo un grave problema por resolver. La motivación de los estudiantes es uno de los grandes condicionantes del éxito escolar y representa un importante papel a la hora de dar respuesta a muchas de las demandas que en cada contexto se plantean a la educación (Anaya, Fernández y Suárez, 2012). Por lo que, los procesos de orientación laboral dirigidos a la transición desde las etapas previas que faciliten la adaptación al bachillerato, los ciclos de formación profesional o la universidad son un objetivo prioritario para promover la continuidad de los estudios en el alumnado. Coincidiendo con Santan, Feliciano y Santana (2013), cabe considerar que la institución escolar ha de favorecer el análisis de las competencias académicas e intereses de los estudiantes para fomentar su madurez, la toma de conciencia y su autonomía en los procesos vocacionales.

La orientación del alumnado ha estado presente en diferentes políticas educativas y de formación para el empleo. A modo de ejemplo, y sin un alcance práctico sustancial, pueden referirse las últimas leyes de educación de nuestro país (LOE, 2006; LOMCE, 2013). En ellas, al menos, se manifiesta cierta sensibilidad y preocupación, concretamente para la etapa de la enseñanza secundaria obligatoria, por el tránsito para la educación postobligatoria y posteriormente, de ser el caso, para la incorporación a la universidad o para el acceso al mundo laboral. Sin embargo, los sistemas de orientación para el asesoramiento profesional, en la práctica, adolecen de un abordaje conjunto o complementario entre las diferentes administraciones (Manzanares, 2013); y la coordinación entre los docentes y otros profesionales colindantes (García-Ruíz, Sánchez y Rodríguez, 2013).

Centrándose la orientación profesional en el proceso de asesoramiento que se desarrolla a lo largo de la vida, cabe considerar desde los contextos de la educación reglada y no reglada su faceta preventiva. Es importante en la orientación y desarrollo de la formación de las personas fomentar su autonomía, el incremento de la capacidad de toma de decisiones y, consecuentemente, su afianzamiento competencial. De hecho, siguiendo a Vélaz-de-Medrano et al. (2012), los orientadores y otros profesionales de la educación valoran su cobertura en los diferentes ámbitos de intervención: orientación personal y académica, tutoría, orientación familiar, atención a la diversidad y, muy especialmente, en la orientación para el tránsito entre etapas educativas y en la orientación académica y profesional del alumnado de la Educación Secundaria Obligatoria (ESO).

Entre otras cuestiones, no puede olvidarse que la escasa madurez o vulnerabilidad de los estudiantes de la etapa de secundaria y la poca información de la que disponen sobre el mundo laboral es susceptible de provocar desconcierto en sus preferencias o de producirle confusión e inestabilidad. Por ello, para Fernández et al. (2007) la orientación profesional representa un papel determinante en el proceso formativo en momentos cruciales, como lo es el del final de la etapa de la escolarización obligatoria. De hecho, uno de los objetivos de la orientación profesional tiene como prioridad ayudar a las personas a tomar conciencia de sus posibilidades y limitaciones académicas para facilitar la toma de responsabilidad y decisión en periodos críticos de su desarrollo, así como de cara a su inserción laboral. Por lo tanto, es deseable y necesario que estos aspectos se aborden desde el proceso formativo.

Otra de las finalidades principales de la orientación profesional en el contexto escolar está encaminada a que las personas desarrollen conductas vocacionales, competencias 
personales y académicas para la toma de conciencia sobre su potencial y expectativas laborales. De este modo, la elección profesional es un proceso de concienciación y toma de decisiones, que se prolonga a lo largo de la vida, mediante el cual cada persona procura constantemente reajustar sus metas a la realidad del mundo del trabajo (Jato y Méndez, 2006). De hecho, la elección académico-profesional es el resultado de un conjunto de variables, esfuerzos, aspiraciones e intereses personales que confluyen durante todo el proceso de evolución, modificables con el tiempo y ante las circunstancias en las que transcurre la existencia humana. Entre algunos de los factores que condicionan esta elección cabe señalar las exigencias del contexto, los recursos disponibles, la autoestima, los intereses, las aspiraciones profesionales y las actitudes. Asimismo, siguiendo a Sepúlveda y Valdebenito (2014) también se encuentran reforzados o aminorados por el nivel de posibilidades con las que cuenta la familia. La literatura vocacional ha puesto de manifiesto tanto el papel de la familia como el de la escuela, los/as compañeros/as y el profesorado (Carvalho y Taveira, 2014); aunque estas líneas de investigación están desarrollada principalmente con estudios de tipología cuantitativa y son escasos los de carácter cualitativo.

La elección profesional y las aspiraciones en la adolescencia pueden encontrarse fuertemente condicionadas, entre otros componentes, por los estereotipos de género. En relación con la toma de decisiones, para la elección de los estudios y las expectativas laborales, la influencia de los estereotipos sexistas resulta históricamente negativa. En este sentido, alguna investigación (Hernández y San Roque, 2006) ha puesto de manifiesto que, tanto en los estudios universitarios, como en los de los ciclos de formación profesional que elige la juventud, están asociados respectivamente con ámbitos tradicionalmente feminizados y masculinizados. Esta misma directriz se mimetiza en lo referente a los intereses y motivaciones laborales.

Cajide et al. (2012) afirman que cuando finaliza el alumnado, de ambos sexos, su formación académica en la enseñanza post-obligatoria (de Bachillerato) le gustaría dedicarse a profesiones como medicina, ingeniería, enfermería, enseñanza, arquitectura e informática. Desglosándolas por género encuentran que, en mucha mayor medida, las adolescentes desean ser enfermeras y médicas; mientras un elevado porcentaje de chicos querrían ser ingenieros, informáticos o profesores. En otros estudios (Ávila y Rocabert, 2001; Manzano, 2004) se sostiene que en la toma de decisiones vocacionales influyen múltiples variables. Entre las mismas, encuentran algunas mediatizadas por estereotipos sexistas en conexión con determinados valores, el autoconcepto, la presión social o las expectativas. De hecho, es notoria la conjugación sexista en las carreras técnicas, a la vez que el papel que continúan ejerciendo las mujeres como madres, atenúa y diversifica su motivación, intereses y aspiraciones.

Con lo expuesto, se pone de manifiesto la relevancia de la variable sexista en las preferencias vocacionales, con todo, también han de considerarse otros elementos, que van muy ligados a este, como son los de carácter contextual y competencial, ya que pueden condicionar o determinar la elección académica y profesional y, en consecuencia, la trayectoria vital de la persona. Coincidiendo con Deaño et al. (2011), la elección vocacional puede estar determinada por diferentes elementos que intervienen en la intersección moldeadora del entorno y deben tenerse en cuenta por la repercusión, tanto positiva como negativa, que pueden ocasionar.

Entre los factores contextuales que pueden condicionar o potenciar la orientación académica y laboral, hay que contemplar la influencia y expectativas de los padres y 
madres, así como las posibilidades socioeconómicas y culturales del entorno. Siguiendo a Vélaz-de-Medrano et al. (2013), cabe considerar los cambios del contexto laboral, que presentan nuevas necesidades ante los sistemas de producción emergentes. Con todo, como cuestión inter-relacionada se ha de apuntar que la selección de los estudios requiere un paso previo en el que el ámbito familiar y los recursos disponibles repercuten. En términos globales, esta influencia en el orden de importancia, después de los progenitores, podría situarse en las personas cercanas a los/as adolescentes: los/as orientadores/as escolares, el profesorado y los familiares próximos.

En relación a la problemática abordada, en el presente trabajo se ha indagado sobre las expectativas y aspectos colindantes que pueden condicionar las preferencias académicas y la tendencia laboral de los/as adolescentes. De modo que con este estudio se trata de conocer el tipo de intereses y las posibles expectativas académicas y laborales que presenta el colectivo. Para ello, como objetivos específicos se plantearon los siguientes:

- Descubrir la motivación, preferencias y hostilidades que encuentra el alumnado en el contexto escolar.

- Identificar las competencias académicas, así como los aspectos que valoran los/as estudiantes y por los que se sienten apreciados/as.

- Indagar sobre los intereses o las expectativas laborales que presenta el alumnado.

- Revelar la afinidad y persistencia existente entre las inclinaciones laborales del alumnado y la profesión de los progenitores.

\section{DISEÑO Y METODOLOGÍA}

Esta investigación se ha abordado con una metodología cualitativa, a través de un estudio de casos múltiples, mediante las narrativas recogidas con un cuestionario de preguntas abiertas. De este modo, el diseño de casos múltiples permite estudiar la realidad a partir de su análisis, descripción y comprensión. Cabe indicar que el trabajo con varios casos refuerza la posibilidad de contrastar los resultados al ampliar el posible número de peculiaridades encontradas (Woodside, 2010).

\subsection{PARTICIPANTES}

Cabe resaltar que las posibilidades de accesibilidad existentes para la recogida de información de tipo narrativo resultan bastante reducidas por la cantidad de dedicación que exige, tanto a los participantes como a los investigadores (Mcmillanm y Shumacher, 2005). Además, teniendo en cuenta la tipología y características de la investigación cualitativa, se ha considerado oportuno abarcar un número comedido de participantes. El estudio se supeditó al alumnado del $4^{\circ}$ curso de la ESO (por tratarse del último curso de la etapa de enseñanza obligatoria en España), considerando tres Institutos de Educación Secundaria (IES) del noroeste de España (para abarcar un estudio de casos múltiples), con una distribución que presentase cierto equilibrio en relación al género.

El número total de alumnado incluido en el estudio fue de 73, implicándose 42 varones y 31 mujeres, y se llevó a cabo en 2014. Estos estudiantes contaban con una edad comprendida entre los 15 y 18 años, presentando una media de 15 años. La distribución por centros, participando la totalidad del alumnado del aula de $4^{\circ}$ curso, ha sido la siguiente: 
- Caso 1 (IES n ${ }^{\circ}$ 1): 30 estudiantes (11 chicas y 9 chicos de 15 años; 3 chicas y 4 chicos de 16 años; y 3 chicos de 17 años).

- $\quad$ Caso 2 (IES n ${ }^{\circ}$ 2): 27 alumnos ( 4 chicas y 3 chicos de 15 años; 6 chicas y 4 chicos de 16; 3 chicas y 6 chicos de 17 años; y un chico de 18 años).

- $\quad$ Caso 3 (IES n ${ }^{\circ}$ 3): 16 adolescentes ( 4 chicas y 9 chicos de 15 años; y 3 chicos de 16 años).

Para preservar el anonimato de los diferentes IES, no se desvela su nombre y en el apartado de resultados, de igual modo, se referencia cada uno como: IES n ${ }^{\circ} 1, \operatorname{IES~n}^{\circ} 2$; e IES no 3 .

\subsection{INSTRUMENTO Y PROCEDIMIENTO DE RECOGIDA DE DATOS}

Como se ha adelantado, el instrumento de recogida de información se utilizó corresponde a un cuestionario de preguntas abiertas que fue elaborado ad hoc para responder a los objetivos de investigación planteada. Para su construcción, inicialmente se esbozaron varios modelos a partir de la consideración de los aspectos formales pertinentes y de la temática objeto de estudio. Posteriormente, se determinó un primer protocolo de cuestiones con dos partes diferenciadas: la primera de ellas dedicada a los datos de identificación y la segunda a las preguntas de contenido.

En la elaboración del cuestionario, siguiendo a Ollaik y Ziller (2012), se han considerado los aspectos de rigor científico clave, sometiéndolo al juicio de expertos en la fase diseño. De este modo, se ha tutelado su construcción y se ha solicitado el consejo de expertos para definir la versión final a partir de la validación de contenido. Este consejo de especialistas se recabó a través de un grupo de discusión en el que participaron 4 investigadores/profesionales. A partir de los mismos se determinó la versión final del instrumento, quedando conformada por la dimensión dedicada a recabar los datos de perfil de los participantes y por otra con 14 preguntas de contenido abierto.

Antes de la aplicación del cuestionario abierto se valoró la conveniencia de suministrarlo digitalmente, decidiendo finalmente administrarlo a través de la plataforma de Gmail en Drive. Esto facilitó una mayor rapidez en la distribución y recogida de datos. Asimismo, el formato online favoreció su cumplimentación de un modo más motivador para el alumnado y ágil para el desarrollo de la investigación.

Para la recogida de información se contactó inicialmente con los directores de los 3 IES, y se consiguió que cediese el profesorado tutor las horas de tutorización académica para este cometido. En el desarrollo de las narrativas, a partir de las preguntas abiertas, los participantes por indicación del profesorado responsable, acudieron al aula de informática del centro, donde una investigadora tenía conectados a Internet los diferentes ordenadores, en el sitio web donde se encontraba ubicado el cuestionario online. De este modo, los implicados pudieron rentabilizar, en mayor medida, el tiempo disponible contando en cada IES con un total de 40 minutos.

Sobre el procedimiento seguido para la recogida de información, también cabe señalar que la investigadora concertó las diferentes citas y se responsabilizó directamente de la toma de datos, dinamizando al alumnado para la producción de las narrativas. De este modo, previamente al suministro de las preguntas le ofreció las consignas pertinentes. Asimismo, en todo momento se mantuvo presencialmente en cada una de las aulas de informática, con total disponibilidad para abordar las posibles dudas de los estudiantes y para realizar las aclaraciones que pudiesen necesitar. 


\subsection{ANÁLISIS DE DATOS}

Las narrativas recogidas a partir de las respuestas proporcionadas por los adolescentes fueron examinadas en su integridad mediante un análisis de contenido. Para ello se utilizó el programa de Analysis of Qualitative Data versión 7 (AQUAD), que permite, a través de la asignación de códigos, la delimitación de las respectivas categorías de análisis y el cómputo de sus frecuencias.

Los análisis de los datos se abordaron por pares de investigadoras, inicialmente a partir de la información bruta obtenida. Para su codificación se partió del asesoramiento de un experto externo, planteándolo en dos pasos: en primer lugar, se acuerda conjuntamente la determinación de la categorización de primer nivel, a partir de la síntesis y fusión de alguno de los enunciados de las preguntas abiertas del cuestionario; mientras que en un segundo momento se demarcaron las de tipo secundario y terciario mediante un proceso inductivo (estas derivaron de las narrativas brindadas por los adolescentes). En una fase posterior se reconfiguraron, con el software de AQUAD, los códigos pertinentes en metacódigos para aunar aquellas subcategorías que presentaban una elevada similitud. Con todo, antes de dar por concluido el proceso de análisis de la información los códigos obtenidos, se han discutido, y posteriormente validado, a partir del juicio de un grupo de expertos externos (que ya se había implicado en el diseño del cuestionario de las preguntas abiertas).

Una vez terminado el análisis a partir de las posibilidades del programa AQUAD, se crearon diferentes tablas de resultados para volcarlas en el de Excel y con ello poder considerar también la preponderancia o singularidad de sus frecuencias (f) en las respectivas subcategorías. Además, una vez transferidos los resultados a Excel, se elaboró su presentación icónica, que resulta de gran utilidad para la descripción, interpretación y comprensión de los mismos.

La garantía de la consistencia interna de los resultados obtenidos se afianzó a través de la sistematización facilitada por el software utilizado. Cabe destacar la rigurosidad que permite en el tratamiento de la información el programa AQUAD. En este sentido, Bensing et al. (2011) subrayan que es necesario garantizar un procedimiento que permita que los datos puedan ser codificados de forma fiel para ser interpretados adecuadamente. Además, para ofrecer evidencias reales sobre la credibilidad de los resultados se aportan diferentes ejemplos textuales. Estos fragmentos se identifican con el número de los respectivos cuestionarios abiertos (CA) y el de la/s línea/s atribuida a las respectivas narrativas en el análisis, la identificación del género del participante y su edad (Ejemplo: CA_063, línea 75-76. Mujer de 17 años).

\section{RESULTADOS}

En este apartado se exponen los resultados principales del estudio realizado, a través de distintos subapartados.

\subsection{MOTIVACIÓN Y DESMOTIVACIÓN EN EL CONTEXTO ESCOLAR}

La motivación por el aprendizaje es un elemento clave que puede repercutir considerablemente sobre la tendencia o ausencia de preferencias vocacionales. En el 
presente estudio los participantes manifestaron diferentes razones que les incentivan para desarrollar el trabajo escolar y algunas que les desmotivan o desaniman. En los respectivos centros analizados coinciden en que lo que más les estimula para acudir al centro educativo es encontrarse e interactuar con sus compañeros o amigos. Además, los estudiantes de los tres IES resaltan el aliciente que les produce el aprendizaje en sí mismo, así como estudiar para poder prosperar. De modo que lo conciben como un medio para un fin. En este sentido se ofrece el siguiente texto:

Lo que más me motiva para estudiar es pensar que gracias a lo que haga ahora en un futuro podré dedicarme a lo que más me gusta (CA_51, línea 6-7. Mujer de 15 años).

Es notorio el interés que muestra el alumnado por la adquisición de recompensas en las distintas asignaturas del currículum (materializadas en la obtención de, al menos, la calificación de aprobado), el disfrute encontrado en el deporte y, en general, con las actividades de ocio. En esta línea se refieren a su participación (durante el recreo) en el equipo de baloncesto de los respectivos centros educativos, al aliciente de que llegue el fin de semana para cambiar la rutina cotidiana y a la posibilidad de disponer de periodos de descanso.

En otro orden de cuestiones, el colectivo analizado indica algunos aspectos que promueven su motivación vinculados con el desarrollo de dinámicas innovadoras en las clases y la generación de un clima de aula agradable. Excepcionalmente, dos alumnos las reducen a obtener la superación del curso académico para promocionar al siguiente. Cabe señalar que, de forma minoritaria, el alumnado del IES $n^{\circ} 1$ y $n^{\circ} 3$ manifiesta que no encuentra dificultades académicas y que nunca solicitó ningún apoyo extra de los/as docentes.

Los resultados obtenidos no revelan globalmente que los/as adolescentes consideren al profesorado como un recurso humano motivacional sustancial. Es más, algunos muestran disconformidad con la actitud que les transmiten distintos/as docentes, en especial los/as del IES $n^{\circ} 3$. Con todo, un pequeño grupo opina que el profesorado puede ejercer diferentes tipos de influencia para que las materias del currículum le resulten más atractivas. Para los/ as participantes de los diferentes centros analizados, el tipo de explicaciones que le ofrecen sus formadores/as les resultan esenciales para facilitarle o proporcionarle el gusto por el contenido de las asignaturas. Aseguran que, dependiendo de la tipología de exposición teórica que le aporten, las clases les pueden resultar interesantes o todo lo contrario.

Una de las características personales que valora positivamente, en particular el alumnado del IES n ${ }^{\circ}$ 2, es el carisma del docente (si desprende simpatía o es ameno en sus explicaciones). Consideran que la actitud positiva del profesorado es muy importante y que en ocasiones su buen humor garantiza que mantengan el interés por la clase. De forma excepcional, algunas alumnas $(\mathrm{f}=3)$ sostienen que el docente ejerce cierta influencia para fomentar el gusto por las asignaturas a partir de un marcado "favoritismo" o de la atención personalizada que les ofrece. Cabe indicar que los estudiantes del IES n 2 recalcan que las clases les provocan cierto aburrimiento. Un grupo elevado de adolescentes ( $\mathrm{f}=41$ ) refleja que la mejor manera de ayudarles de parte del profesorado en el proceso de aprendizaje, en las distintas asignaturas del currículum, es mediante el refuerzo académico a partir de una enseñanza individualizada (presionándoles y facilitándole técnicas de estudio).

En el alumnado del IES n ${ }^{\circ} 2$ sobresalen, en relación a la terminación de los estudios, aspectos que no se advierten en los demás: el sentido de valoración de los mismos, y la 
proyección que le confieren para el futuro. Sin embargo también se detectan otras posturas contrarias. Por ejemplo, a una alumna no le motiva ninguna actividad o situación vinculada con el contexto escolar (declarando que asiste al centro porque está obligada por sus padres); mientras, en otro orden de cuestiones, un chico sostiene que le agrada madrugar todos los días para aprovechar la mañana y realizar trabajos útiles. Sin embargo, es este último aspecto uno de los que menos les agrada a los/as estudiantes de los tres institutos analizados. De este modo, se pone de manifiesto que levantarse temprano para acudir al aula es lo que peor llevan, en relación al ámbito escolar, en particular el alumnado del IES $\mathrm{n}^{\mathrm{o}} 1$ (5 de los 16 participantes). Véanse los ejemplos siguientes:

Lo que menos me gusta es tener que ir al colegio tan temprano diariamente y no poder descansar lo necesario" (CA_032, líneas 11-13. Hombre de 15 años).

Lo que más me motiva es estudiar para conseguir el día de mañana un trabajo remunerado y cómodo (CA_055, líneas 14-16. Mujer de 15 años).

Tabla 1. Relación entre asignaturas preferidas y hostiles por género

\begin{tabular}{lcccc}
\hline \multicolumn{1}{c}{ Género } & Mujer & Hombre & Mujer & Hombre \\
\hline \multicolumn{1}{c}{ Asignaturas } & \multicolumn{2}{c}{ Preferidas } & \multicolumn{2}{c}{ Hostiles } \\
\hline Artísticas & 8 & 12 & 0 & 0 \\
CC EE & 6 & 18 & 20 & 6 \\
CC SS & 7 & 2 & 3 & 3 \\
Lenguas & 18 & 7 & 8 & 20 \\
Otras & 5 & 22 & 9 & 8 \\
\hline
\end{tabular}

En relación a las asignaturas favoritas y las que le producen hostilidad al alumnado, por género (Tabla 1), los resultados ponen de manifiesto que las materias del currículum que encuentran más arduas las chicas son las de Ciencias Experimentales (CC EE) y los chicos las de Lenguas: lengua y literatura española; lengua y literatura gallega y lengua extranjera, en especial la inglesa (mostrando, tajantemente, algunos estudiantes, el rechazo por el aprendizaje del inglés). En contraposición, las materias favoritas de las alumnas son las de Lenguas, mientras que los varones presentan una predilección por las de CC EE. Sin embargo, por otras materias curriculares (como informática y tecnología) existe un gran interés entre los adolescentes hombres, mientras la preferencia en las chicas es notablemente menor.

\subsection{COMPETENCIAS, VALORES Y PREFERENCIAS LABORALES}

Globalmente, se puede determinar que los y las adolescentes destacan por sus competencias de tipo cognitivo y las asocian con la retención de contenidos, memorización, comprensión y capacidad de redacción. Enlazado con los estereotipos sexistas, se detecta que las habilidades en las que piensan que sobresalen algunos chicos son las de tipo deportivo, 
asociándolas con: jugar al baloncesto, hacer gimnasia, practicar deporte de riesgo o de resistencia física. Cabe señalar que en las capacidades de carácter artístico son las adolescentes quienes consideran que disponen de las vinculadas con el dibujo, música y pintura. En cuanto a las que conectan con aspectos referidos a responsabilidades, excepcionalmente dos adolescentes (mujeres) sostienen que recaen más en las chicas, al igual que las de cercanía o las relacionadas con los factores humanos. A modo de ejemplo, se rescata el texto siguiente:

Creo que se me da bien escuchar y explicar a los demás las cosas, además de que somos bastante responsables las chicas en comparación con nuestros compañeros (CA_006, línea 107-109. Mujer de 15 años).

Tanto los chicos como las chicas, independientemente del género expresan que se sienten valorados/as por diferentes aspectos intrínsecos. Entre estos componentes incluyen la amabilidad, capacidad de comprensión o escucha, honestidad, sinceridad, sociabilidad y solidaridad. Por otra parte, como atributos externos puntualizaron algunas cuestiones asociadas con el aspecto físico y económico. A modo de ejemplo se ofrecen los siguientes extractos:

Por una parte por mi personalidad, soy muy simpático, quiero bien a mis amigos, soy muy meticuloso y me intereso por muchas cosas y nunca dejaría que le hiciesen daño a mis compañeros o a la gente cercana a mí (CA_031, líneas 56-60. Hombre de 15 años).

Primero me valoran por el físico, ya que la gente antes de conocerte siempre se fija en eso y después me valoran por mi forma de ser (CA_006, línea 54-57. Mujer de 15 años).

De forma congruente con lo que valoran de los demás, los/as adolescentes también aprecian en otras personas, independientemente del género, sus cualidades intrínsecas (a excepción de una chica y un chico). Entre los componentes de índole interna, estiman de los demás su amabilidad, apoyo y ayuda manifestada, muestras de bondad, manifestación de confianza, de generosidad, honestidad, lealtad, respeto, capacidad de escucha, simpatía y sinceridad. Como aspectos extrínsecos, se han referido al físico y al nivel económico fundamentalmente.

Figura 1. Relación entre ocupaciones, género y valores

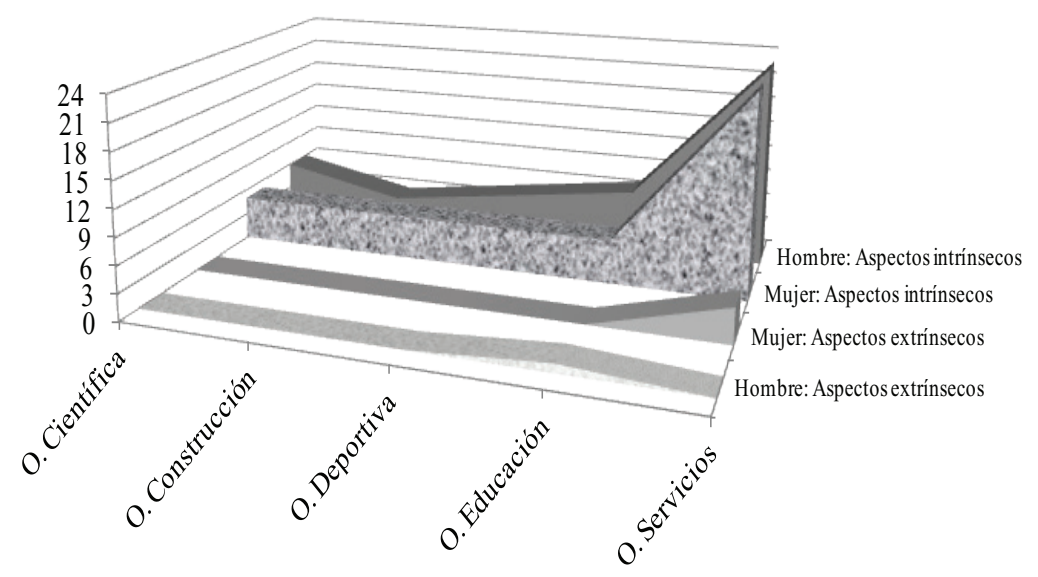


A partir de la preferencia que manifiestan los y las adolescentes, al margen de la variable del género, las ocupaciones $(\mathrm{O})$ relacionadas con los servicios (antidisturbios, camarero/a, comercial, hostelera/o, mecánico, policía y marketing) son elegidas por participantes que valoran en las demás personas, primordialmente sus cualidades intrínsecas (Figura 1). Con todo, también cabe indicar que un pequeño grupo que realza las características externas también se inclina por ocupaciones relacionadas con los servicios. Los que valoran los aspectos extrínsecos no enfatizan la preferencia por ningún tipo de profesión.

Tanto las chicas como los chicos que destacan los aspectos internos de las personas se inclinan, en segundo lugar, por ocupaciones científicas (relacionadas con las materias de biología o física y química), acentuándose esta tendencia en las adolescentes. Por otro lado, las profesiones conectadas con la educación (profesora de infantil, de secundaria, de educación física, de conservatorio) y trabajo social les resultan más atractivas a las mujeres. Mientras que las relacionadas con los deportes o la gimnasia (jugador de baloncesto, de futbol profesional o entrenador personal) son las preferidas por los varones. Indicadores que continúan demostrando el mantenimiento de una tendencia sexista como una dominante histórica.

\subsection{DEMANDAS LABORALES Y AFINIDAD CON LAS DE LOS PROGENITORES}

A las adolescentes mujeres (M) les resultan más atractivas las ocupaciones de tipo sanitario (Figura 2). En estas incluyen las de dentista, veterinaria, fisioterapia, microbiología, medicina y enfermería. Por otra parte, algunas apuntan las concernientes a la educación. Otras ocupaciones $(\mathrm{O})$ que presentan un elevado nivel de interés para el alumnado de secundaria, tal como ya manifestaron en sus preferencias laborales, independientemente del género, son las del sector servicios. Entre estas se refieren a las vinculadas con empresariales y turismo, diseño y hostelería. A modo de ejemplo, se presenta el siguiente extracto:

Las profesiones más interesantes para mí son las de policía, hostelería, sanidad y profesor porque ayudas a la gente y son útiles para todos (CA_066, líneas 69-71. Mujer de 16 años).

Figura 2. Preferencias profesionales por género

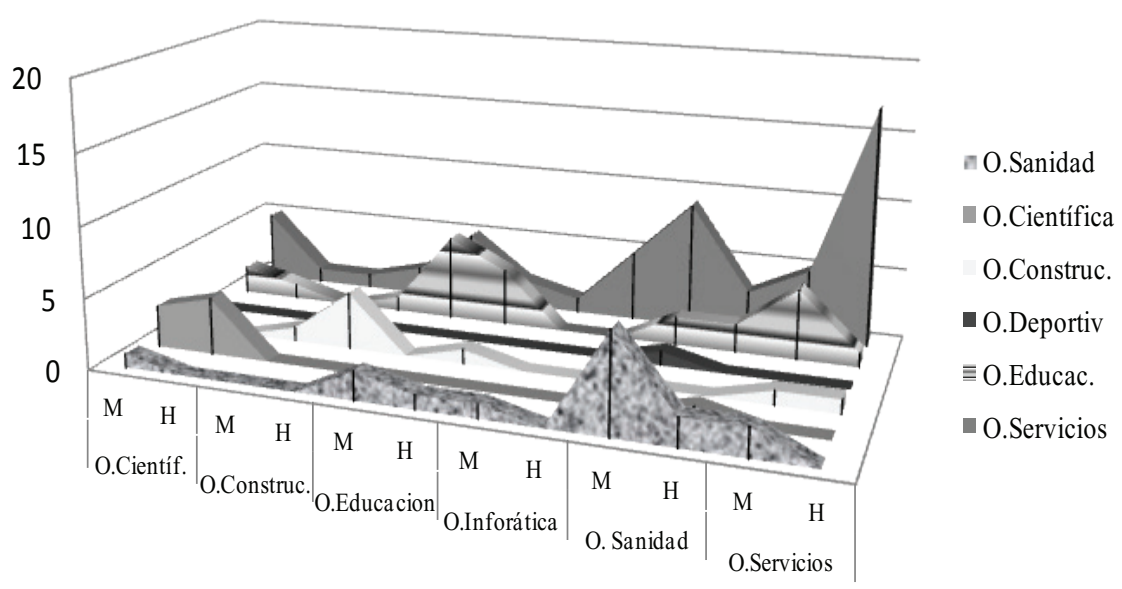


En las profesiones que integran aspectos relacionados con la informática (diseñador de videojuegos y programación informática) los hombres $(\mathrm{H})$ expresan una inclinación acusada, mientras en las chicas se observa una baja incidencia. Del mismo modo, por las ocupaciones concernientes a la construcción (albañilería y arquitectura) y las de tipo científico (astronomía, biología, química, etc.), los adolescentes (varones) muestran una mayor preferencia. Una evidencia del interés expresado por las mujeres se percibe en el siguiente texto:

La más interesante, por ejemplo, me parece la de ser enfermera porque ayudas a la gente y es más fácil que tengan trabajo (CA_063, línea 75-76. Mujer de 17 años).

Se descubre un punto de unión entre las profesiones anheladas y las que le resultan más interesantes, apuntando a las del sector servicios (Figura 2). Hecho que se revela notorio en el caso de los varones $(\mathrm{f}=17)$. Con una menor incidencia, esto mismo se detecta en 5 mujeres. Otro dato a considerar es que las profesiones relacionadas con la educación les parecen interesantes a un grupo de chicas y desearían dedicarse a ellas en el futuro. En el caso de los chicos únicamente cuatro coinciden en esta preferencia.

A partir de los resultados obtenidos, se encuentran diferentes coincidencias en las profesiones de los padres y madres con las deseadas por los/as adolescentes (tabla 2). En las referidas al sector servicios (administración, mecánica y hostelería) se detectó la inclinación por las mismas en 11 chicas y 18 chicos. Además, comparando la profesión de la madre con la deseada por los y las participantes se encuentra conexión en las ocupaciones asociadas con los servicios, de manera casi igualada para ambos géneros ( $\mathrm{f}=17$ en las mujeres y $\mathrm{f}=18$ en los varones).

Tabla 2. Concordancia entre la profesión de los/as progenitores y la demandada por el alumnado

\begin{tabular}{|c|c|c|c|c|c|c|c|c|}
\hline Género & $\mathrm{M}$ & $\mathrm{H}$ & $\mathrm{M}$ & $\mathrm{H}$ & $\mathrm{M}$ & $\mathrm{H}$ & $\mathrm{M}$ & $\mathrm{H}$ \\
\hline $\begin{array}{l}\text { Profesión } \\
\text { del padre }\end{array}$ & \multicolumn{2}{|c|}{ Construcción } & \multicolumn{2}{|c|}{ Sanitaria } & \multicolumn{2}{|c|}{ Servicios } & \multicolumn{2}{|c|}{ Ingeniería } \\
\hline Científica & 0 & 1 & 0 & 2 & 4 & 1 & 0 & 0 \\
\hline Construcción & 0 & 2 & 1 & 0 & 1 & 3 & 0 & 0 \\
\hline Deporte & 0 & 1 & 0 & 0 & 4 & 5 & 0 & 0 \\
\hline Educación & 2 & 1 & 0 & 0 & 5 & 4 & 2 & 0 \\
\hline Servicios & 2 & 8 & 0 & 1 & 11 & 18 & 1 & 0 \\
\hline $\begin{array}{l}\text { Profesión } \\
\text { de la madre }\end{array}$ & \multicolumn{2}{|c|}{ Educación } & \multicolumn{2}{|c|}{ Sanitaria } & \multicolumn{2}{|c|}{ Servicios } & & \\
\hline Científica & 0 & 0 & 1 & 2 & 3 & 2 & & \\
\hline Construcción & 0 & 0 & 0 & 0 & 2 & 4 & & \\
\hline Deportiva & 0 & 1 & 0 & 1 & 4 & 5 & & \\
\hline Educación & 3 & 0 & 0 & 0 & 5 & 7 & & \\
\hline Servicios & 1 & 1 & 1 & 7 & 17 & 18 & & \\
\hline
\end{tabular}


Al margen de alguna excepción, los resultados reflejan que los participantes defienden su pronunciada continuidad en el tiempo sobre los intereses de la tendencia profesional. Observándolos por centros, también se plasma de forma notoria la estabilidad sostenida, en particular cabe señalar los del IES $n^{\circ} 1$, al argumentarlo concienzudamente 13 adolescentes (4 chicas y 9 chicos) frente a los 5 que se muestran influenciables a las ideas de otras personas. Estas posibles alteraciones las expresan independientemente del género. A modo de ejemplo, véanse dos ejemplos contrapuestos:

A mí las ideas de los otros me dan igual, porque cada uno debe de tener su personalidad y creer en sí mismo, y si a uno le gusta una profesión, por los motivos que sea, nadie le tiene que hacer cambiar de idea, sea por lo que sea, es tu decisión, yo por lo menos no la cambiaré (CA_032, línea 97-102. Hombre de 15 años).

Mis preferencias en los estudios van cambiando con el tiempo, aun no tengo una idea fija de lo que quiero ser en el futuro porque tampoco me paré a pensarlo mucho (CA_001, línea 59-62. Mujer de 15 años).

\section{DISCUSIÓN Y CONCLUSIONES}

A cada adolescente, en el momento de decidir, conviene que tenga lo más claro posible cuáles son las metas que pretende y cómo obtener los logros para afrontar con éxito su futura profesión o, de ser el caso, sus estudios posteriores de continuidad. Por ello es importante que, al menos, el profesorado y sus progenitores conozcan el tipo de alicientes que les empujan o la posible desmotivación que experimentan. En este sentido, a partir de los resultados obtenidos puede concluirse que el colectivo de adolescentes tiene como principal motivación para asistir a las clases en el centro educativo la interacción con sus compañeros/as o amigos/as, así como el horizonte de terminar los estudios de la enseñanza obligatoria. Lo que menos les motiva es madrugar, por lo que sería recomendable plantear algún posible cambio en el horario, al menos, a modo de gesto de complicidad, algún día de la semana. Es esencial incluir medidas que refuercen la motivación del alumnado cuando, entre otros autores, Marsh (2012) destaca su trascendencia como factor desencadenante del éxito académico.

En este estudio se detecta que las estrategias motivacionales más valoradas por el alumnado, y también las más utilizadas por el profesorado, son las asociadas con el refuerzo educativo. Por su parte, Suárez y Fernández (2013) resaltan las que contribuyen a establecer relaciones personales sólidas, que junto a las de tipo cognitivo favorecen la autorregulación metacognitiva. Vans y Ridder (2003) sostienen que entre los componentes motivacionales supeditados a la profesión docente destacan diferentes deficiencias pedagógicas fruto de la poca claridad en la presentación de la materia, el no promover la reflexión ni el juicio crítico, un parco conocimiento científico de la disciplina impartida y la falta de vocación docente.

En cuanto a las asignaturas preferidas, para las chicas hay que referir las de letras; mientras los varones se decantan en mayor medida por las de informática y tecnología. En el extremo opuesto, las materias que les resultan más hostiles a los adolescentes (varones) son las asociadas con las lenguas; sin embargo las mujeres sienten una mayor resistencia por las de CC EE (matemáticas, biología y física-química). Por otro lado, es de indicar que los estudiantes consideran que el profesorado cuenta con una influencia notoria para 
impulsar o aplacar sus preferencias académicas. Como principales factores de "persuasión" en el aprendizaje, el alumnado se refiere a los aspectos competenciales del docente (por ejemplo, los asociados con la capacidad para facilitarle las explicaciones académicas) o los rasgos de personalidad, que facilitan el acercamiento o contribuyen a generar una mayor empatía.

De esta investigación se desprende que los chicos destacan en competencias ligadas a las habilidades de tipo deportivo y las chicas con las de carácter cognitivo (en particular asociadas con las lenguas) y artes. Al hilo de esta cuestión, en un estudio de Moreno y Pavón (2008) se pone de manifiesto que la mayoría de los adolescentes creían que se le daba bien la práctica deportiva, mientras que alrededor de un tercio de las mujeres consideraban que no tenían posibilidades. Por otro lado, Echavarri, Godoy y Olaz (2007) detectaron que las jóvenes aventajan a los varones en pruebas de fluidez verbal, lo que reafirma el hecho de que las chicas destaquen por sus habilidades cognitivas.

Es de considerar, de cara a la toma de conciencia en la elección profesional, la importancia que otorgan los/as adolescentes a los valores. En este sentido el colectivo ha puesto de manifiesto que aprecia en los demás, principalmente, sus cualidades interiores. Lo mismo ocurre con lo que creen que valoran en ellas y ellos otras personas. Este hallazgo refleja un cambio de tendencia, cuando en un estudio anterior (Rivas, 2007) se apuntó que respecto a la preferencia laboral además del sexo/género, les influyen fundamentalmente cuestiones relacionadas con la posibilidad de adquirir dinero y poder (todas enfatizaban los aspectos externos).

De forma extensiva se puede sostener que, entre otras, el colectivo de adolescentes enlaza las expectativas sobre las preferencias profesionales con los valores e intereses personales y contextuales. De esta investigación se desprende que los chicos y las chicas que consideran importantes las cualidades personales, se interesan por profesiones relacionadas con los servicios. Por su parte, la mayoría de los padres y madres de los participantes pertenecen a este mismo sector. En cuanto a la correspondencia de la profesión de sus progenitores y a la que les gustaría dedicarse, se puede avalar que coinciden también con la del sector servicios, indistintamente de la ejercida por su padre o madre; mientras otro estudio años atrás (Estrada, 2009) se ha referido a una cierta influencia de la madre. Por su parte, Cepero (2009) apuntó que las chicas eligen ramas típicamente femeninas ligadas a los servicios, cuando los chicos prefieren las relacionadas con la industria, la electrónica y el metal. Con todo, a partir de este trabajo se concluye, independientemente del género, que a los y las adolescentes les gustaría dedicarse mayoritariamente a una profesión relacionada con el sector servicios. Este hallazgo presenta globalmente un avance positivo al detectar que se produce una tendencia progresista con la difuminación de las marcas características de tipo sexista que se han acuñado históricamente en las preferencias y roles profesionales.

En cuanto a la persistencia de los intereses o expectativas profesionales los/as participantes aseguran con rotundidad la firmeza en los mismos. De lo que se deduce que son poco influenciables por las ideas de los demás. En este sentido, cabe traer a colación que cuando los progenitores suponen un apoyo esencial para fortalecer la tenacidad de los hijos e hijas ante situaciones adversas, generan una influencia positiva en el proceso de autorregulación del rendimiento académico y en general de las expectativas (Suárez et al., 2011). Si esto, a su vez, se refuerza con una adecuada dosis de confianza desde el contexto familiar y escolar los resultados mejorarían previsiblemente de manera considerable. 


\section{REFERENCIAS BIBLIOGRÁFICAS}

Anaya, N., Fernández, A. P., \& Suárez, J. M. (2012). Niveles motivacionales en los estudiantes de secundaria y su discriminación en función de las estrategias motivacionales. Revista Española de Orientación y Psicopedagogía, 23(1), 50-65.

Ávila, V., \& Rocabert, E. (2001). Características vocacionales de estudiantes universitarios ciegos y deficientes visuales: una aproximación cualitativa. Revista Española de Orientación y Psicopedagogía, 22(12), 319-340.

Bensing, J., Deledda, G., Fletcher, I., Mazzi, M., Moretti, F., Rimondini, M., \& Van Vliet, L. A. (2011). Standardized approach to qualitative content analysis of focus group discussions from different countries. Patient Education \& Counseling, 82, 420- 428.

Cajide, J., Castro, M. D., Mosteiro, M. J., Porto, A., Sierra, S., \& Sobrino, M. E. (2012). Expectativas de éxito y percepción de competencias en ciencia y tecnología en alumnas y alumnos de bachillerato. Recuperado desde: http://www.oei.es/congresoctg/memoria/pdf/2PortoCastro.pdf

Carvalho, M., \& Taveira, M. C. (2014). El papel del profesorado en la toma de decisiones académico y profesionales del alumnado. Revista Española de Orientación y Psicopedagogía, 25(3), 20-35.

Cepero, A. B. (2009). Las preferencias profesionales y vocacionales del alumnado de secundario y formación profesional específica. Granada: Universidad de Granada.

Deaño, M., Tellado, F., García-Señorán M. M., y Brandín, C. (Eds.). (2011). Modelos de orientación e intervención psicopedagógica. Ourense: Ediciones GERSAM.

Echavarri, M., Godoy, J. C., \& Olaz, F. O. (2007). Diferencias de género en habilidades cognitivas y rendimiento académico en estudiantes universitarios. Universitas Psychologica, 6(2), 319-330.

Estrada, P. (2009). Factores que intervienen en la elección de carrera de estudiantes de bachillerato de dos modalidades educativas. Recuperado desde: http://www.comie.org.mx/congreso/ memoriaelectronica/v11/docs/area_16/2028.pdf

Fernández, C., Peña. J. V., Viñuela, M. P., \& Torío, S. (2007). Los procesos de orientación escolar y la toma de decisiones académica y profesional. Revista Complutense de Educación, 18(2), 87-103.

García-Ruíz, R., Sánchez, A., \& Rodríguez, A. (2013). Las competencias socio-personales en la integración socio-laboral de los jóvenes que cursan programas de cualificación profesional inicial. Revista Española de Orientación y Psicopedagogía, 24(2), 58-78.

Hernández, V., \& San Roque, I. (2006). Ciclos formativos 2005. Preferencias de los estudiantes de la comunidad de Madrid. Madrid: Universidad Pontificia de Comillas.

Jato, E., \& Méndez, M. J. (Coords.). (2006). Elixir o futuro sen prexuízos. Santiago de Compostela: Tórculo.

LOE. (2006). Ley Orgánica 2/2006, de 3 de mayo, de Educación. Boletín Oficial del Estado (de 4 de mayo de 2006), n.106.

LOMCE (2013). Ley Orgánica 8/2013, de 9 de diciembre, para la mejora de la calidad educativa. Boletín Oficial del Estado (de 10 de diciembre de 2013), n. 295.

Manzanares, A. (2013). Sistemas integrados de orientación. Una propuesta para la articulación de estructuras y servicios a nivel local. Revista Española de Orientación y Psicopedagogía, 24(1), 62-77.

Manzano, G. (2004). Burnout y Engagement. Relación con el desempeño, madurez profesional y tendencia al abandono de los estudiantes. Revista de Psicología Social, 23(1), 69-75.

Marsh, H. (2012). Relationships for learning: Using pupil voice to define teacher-pupil relationships that enhance pupil engagement. Management in Education, 26(3), 161-163.

Mcmillan, J., \& Schumacher, S. (2005). Investigación educativa: una introducción conceptual. Madrid: Pearson.

Moreno, J. A., \& Pavón, A. (2008). Actitud de los universitarios ante la práctica físico deportiva: diferencias de géneros. Revista de Psicología del Deporte, 17(1), 7-23.

Ollaik, L. G., \& Ziller, H. M. (2012). Concepções de validade em pesquisas qualitativas. Educação 
e Pesquisa, 38(1), 229-241.

Rivas, F. (2007). ¿Conducta y asesoramiento vocacional en el mundo de hoy? Revista Electrónica de Investigación Psicoeducativa, 5(11), 5-14.

Santana, L. E., Feliciano, L. A., \& Santana, J. A. (2013). Madurez y autoeficacia vocacional en $3^{\circ}$ y $4^{\circ}$ de ESO, bachillerato y ciclos formativos. Revista Española de Orientación y Psicopedagogía, 24(3), 8-26.

Sepúlveda, L., \& Valdebenito, M. J. (2014). ¿Las cosas claras? Aspiraciones de futuro y proyecto educativo laboral de jóvenes estudiantes secundarios. Estudios Pedagógicos, 40(1), 243-261.

Suárez, J. M., \& Fernández, A. P. (2013). Un modelo sobre cómo las estrategias motivacionales relacionadas con el componente de afectividad inciden sobre las estrategias cognitivas y metacognitivas. Educación XX1, 16(2), 231-246.

Suárez, N., Tuero-Herrero, E., Bernardo, A., Fernández, M., Cerezo, R., González-García, J.A., Rosario, P., \& Núñez, J. C. (2011). El fracaso escolar en educación secundaria: análisis del papel de la implicación familiar. Revista de Formación del Profesorado e Investigación Educativa, 24, 49-64.

Vans, J. C., \& Ridder, G. (2003). Fast track or failure: a study of the graduation and dropout rates of Ph. D. students in economiscs. Economics of Education Review, 22(2), 157-166.

Vélaz-de-Medrano, C., Blanco-Blanco, A. y Manzano-Soto, N. (2012). Cobertura de necesidades de orientación y tutoría en la educación obligatoria: estudio en nueve comunidades autónoma. Revista de Educación, Número extraordinario, 138-173.

Vélaz-de-Medrano; C., Manzanares, A., López-Martín, E., \& Manzano-Soto, N. (2013). Competencias y formación de los orientadores escolares: estudio empírico en nueve comunidades autónomas. Revista de Educación, Número extraordinario, 261-292.

Woodside, A. G. (2010). Case study research: theory, methods, practice. Bingley: Emerald. 
